\title{
Diagnóstico del nivel de sostenibilidad de las empresas del Sector Gastronómico del municipio de Olocuilta
}

\author{
Por. \\ Ileana Aguirre, Nathaly Castaneda, José Cornejo, José González, Laura Orellana
}

\begin{abstract}
RESUMEN
Una empresa sostenible es aquella que busca crear valor medioambiental, social y económico a corto, mediano y largo plazo, buscando así un impacto positivo al bienestar y al progreso de las presentes y futuras generaciones. El diagnóstico del nivel de sostenibilidad en las empresas del sector gastronómico del municipio de Olocuilta, pertenecientes específicamente al pupusódromo "El Manguito", tiene como objetivo evaluar las micro y pequeñas empresas, con base en indicadores generalizados orientados a medir el nivel de sostenibilidad actual de las mismas. Se realizó una investigación de carácter exploratorio, en la cual se emplearon encuestas y se realizaron entrevistas, se recopiló información referente a los tres factores que integran el marco de la sostenibilidad. Los resultados obtenidos demuestran que existe una gran fortaleza a nivel económico y oportunidades de mejora social y medioambiental.
\end{abstract}

Palabras claves: sostenibilidad, indicador, ratio, parámetro.

\section{Introducción}

El nivel de sostenibilidad en las empresas se encuentra directamente relacionado con la interacción de los tres factores que la integran. Es decir, se busca crear beneficios económicos para la empresa, minimizando el impacto negativo en el medio ambiente causa- do por las prácticas productivas y de forma paralela aportando beneficios y asegurando condiciones idóneas al área social.

Alcanzar un buen nivel de sostenibilidad presenta un gran reto para las empresas debido a que se necesita un plan de acción bastante elaborado y en algunos casos de una inversión alta.

Al comparar los indicadores económicos con base en la producción o inversión con los indicadores ambientales a lo largo del tiempo se observa un comportamiento contrario. Es decir, mientras los económicos resultan a lo largo del tiempo cada vez más positivos, los ambientales presentan una tendencia negativa cada vez más definida, manifestándose en una contaminación sin fronteras y un cambio climático el cual degrada los ecosistemas, afectando la biodiversidad y atentando contra la supervivencia humana. Existe un estudio realizado por Meadows llamado "Los límites del crecimiento" donde se describe la vinculación entre ambos tipos de indicadores lo cual genera la posibilidad de un crecimiento sostenido e indefinido y determina la necesidad de un desarrollo sostenible (Vilches, 2014).

En el año de 1999 se crean los 10 principios de ciudadanía corporativa, emitidos por el Pacto Mundial de las Naciones Unidas, los cuales aportan a la sostenibilidad empresarial en un marco para garantizar la responsabilidad social corporativa (Gualdrón, 2018).
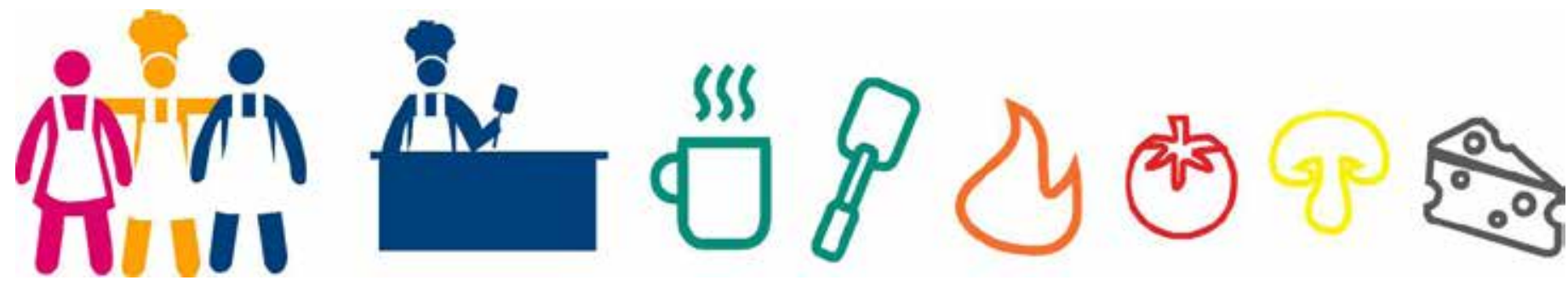
En el año de 1999 se crean los 10 principios de ciudadanía corporativa, emitidos por el Pacto Mundial de las Naciones Unidas, los cuales aportan a la sostenibilidad empresarial en un marco para garantizar la responsabilidad social corporativa (Gualdrón, 2018). Dichos principios son:

a. Las empresas deben apoyar la protección de los derechos humanos fundamentales reconocidos internacionalmente dentro de su ámbito de influencia. Ya que son de vital importancia para la organización, los grupos de interés y la comunidad, públicamente se reconoce que el respeto a los derechos humanos contribuye a mejorar la producción de las empresas.

b. Las empresas deben asegurarse de no ser cómplices en la vulneración de los derechos humanos. Procurando generar condiciones dignas, tanto en el ámbito laboral como en el social promoviendo así que en la cadena de valor no se genere violación a los derechos humanos.

c. Las empresas deben apoyar la libertad de afiliación y el reconocimiento efectivo del derecho a la negociación colectiva, con sindicatos, asociaciones y participación de los trabajadores en las decisiones corporativas.

d. Las empresas deben apoyar la eliminación de toda forma de trabajo forzoso o realizado bajo coacción.

e. Las empresas deben apoyar la erradicación del trabajo infantil.

f. Las empresas deben apoyar la abolición de las prácticas de discriminación en el empleo y la ocupación. Logrando así la inclusión de jóvenes, mujeres y adultos.

g. Las empresas deben tener un enfoque preventivo que favorezca el medio ambiente.

h. Las empresas deben fomentar las iniciativas que promuevan una mayor responsabilidad ambiental.

i. Las empresas deben favorecer el desarrollo y la difusión de tecnología respetuosa con el medio ambiente. j. Las empresas deben trabajar contra la corrupción en todas las formas incluidas extorsión y soborno. Las empresas contribuyen a reducir riesgos, las hace eficientes y mejor posicionadas.

Por lo expuesto anteriormente, el diagnóstico orientado a medir el nivel de Sostenibilidad que presentan las empresas pertenecientes al pupusódromo "El Manguito", evalúa la situación actual de los tres factores que integran el nivel de sostenibilidad por medio de indicadores que poseen las empresas.

\section{Metodología}

Se realizó una investigación de carácter exploratorio en el mes de junio de 2019. Las variables o subindicadores para evaluar la sostenibilidad empresarial son extraídas de un estudio previo "Identificación de indicadores que caracterizan una micro y pequeña empresa", por Alas Henríquez, Avilés Aguilar, \& Burgos Villatoro en el año 2018.

En dicho estudio se establece una estructura para la formulación de indicadores de sostenibilidad; a partir de algunas propuestas específicas: el modelo de indicadores de Responsabilidad Social Corporativa (RSE) para Pequeña y Mediana empresa (PYMES; la medición de la sustentabilidad en una empresa teniendo en cuenta el cumplimiento de los objetivos estratégicos de la empresa respecto a diversos factores internos y herramientas para medir la sostenibilidad corporativa, la cual consta de herramientas que evalúan el grado de avance o logro de las metas establecidas de una empresa.

Con dicha metodología, los autores proponen 14 indicadores de sostenibilidad que toman en cuenta factores como: la rentabilidad financiera, liquidez financiera, gestión de actividad financiera, el ambiente de trabajo, infraestructura, ergonomía, relación con el trabajador, programa de relación con la comunidad, gestión de relación con proveedores y clientes, calidad de productos y servicios, programa de gestión medioambiental, consumo de energía, disposición de residuos sólidos y consumo total de energía; de forma que al evaluarlos se puede determinar si una empresa es o no sostenible.

El cumplimiento de los indicadores previamente mencionados son los que determinan la sostenibilidad de una empresa. Sin embargo, la aplicación de estos dependerá directamente del rubro al cual pertenece la empresa. 
Para el rubro de negocio evaluado en este estudio, sector gastronómico, los indicadores y subindicadores a evaluar se encuentran detallados en la Tabla 1.

Tabla 1. Subindicadores económicos, sociales y ambientales

\begin{tabular}{|c|c|c|}
\hline FACTOR & INDICADOR & SUBINDICADOR \\
\hline \multirow{3}{*}{ ECONÓMICO } & Rentabilidad financiera & $\begin{array}{l}\text { Rentabilidad neta del activo } \\
\text { Margen bruto } \\
\text { Margen ocupacional } \\
\text { Rentabilidad operacional del patrimonio }\end{array}$ \\
\hline & Liquidez financiera & $\begin{array}{l}\text { Liquidez financiera } \\
\text { Prueba ácida } \\
\text { Prueba defensiva } \\
\text { Capital de trabajo }\end{array}$ \\
\hline & $\begin{array}{l}\text { Gestión de actividad } \\
\text { financiera }\end{array}$ & $\begin{array}{l}\text { Rotación de cartera } \\
\text { Rotación de inventarios } \\
\text { Rotación medio de pago a proveedores } \\
\text { Rotación de cajas y bancos } \\
\text { Rotación de activos totales } \\
\text { Rotación de activos fijos }\end{array}$ \\
\hline \multirow{5}{*}{ SOCIAL } & Ambiente de trabajo & Evaluación de riesgo ocupacional \\
\hline & Infraestructura & Porcentaje de accidentes por fallas en la infraestructura \\
\hline & $\begin{array}{l}\text { Relación con } \\
\text { el trabajador }\end{array}$ & $\begin{array}{l}\text { Salario minimo de la empresa en comparación al de la ley } \\
\text { Prestaciones sociales de acuerdo a la ley } \\
\text { Beneficios sociales extra a los requeridos por la ley } \\
\text { Programa para escuchar sugerencias, quejas y } \\
\text { reclamos del trabajador } \\
\text { Reunión de la gerencia con los trabajadores } \\
\text { para informar sobre actualizaciones de la empresa } \\
\text { Porcentaje de conflictos laborales que se han resuelto } \\
\text { Rotación de personal }\end{array}$ \\
\hline & $\begin{array}{l}\text { Gestión de relación } \\
\text { con proveedores y clientes }\end{array}$ & $\begin{array}{l}\text { Existencia de un sistema de comunicación con el cliente } \\
\text { Porcentaje de quejas y reclamos } \\
\text { Existencia de un plan de evaluación formal para proveedores } \\
\text { Porcentaje del personal capacitado sobre atención a clientes. }\end{array}$ \\
\hline & $\begin{array}{l}\text { Calidad de productos } \\
\text { y servicios }\end{array}$ & $\begin{array}{l}\text { Existencia de un plan de gestión de calidad } \\
\text { Existencia de un servicio postventa o } \\
\text { retroalimentación para clientes } \\
\text { Existencia de un plan de mejora continua }\end{array}$ \\
\hline \multirow{4}{*}{ AMBIENTAL } & $\begin{array}{l}\text { Programa de gestión } \\
\text { medio ambiental }\end{array}$ & $\begin{array}{l}\text { Nivel de conocimiento del tema medioambiental } \\
\text { por parte de directivos y empleados } \\
\text { Porcentaje de inversión consignado a programas que } \\
\text { eviten el impacto medioambiental producido } \\
\text { por la actividad económicos } \\
\text { Nivel de daño generado por los medios de transporte } \\
\text { utilizados por la logistica y la cadena de suministro }\end{array}$ \\
\hline & Consumo de agua & $\begin{array}{l}\text { Consumo de Agua } \\
\text { Porcentaje utilizado para producción del total de consumo } \\
\text { Porcentaje utilizado para consumo humano del total de consumo } \\
\text { Porcentaje total de vertido liquido }\end{array}$ \\
\hline & $\begin{array}{l}\text { Disposición de } \\
\text { residuos solidos }\end{array}$ & $\begin{array}{l}\text { Total de residuos producidos por la empresa } \\
\text { Porcentaje de residuo reciclado }\end{array}$ \\
\hline & $\begin{array}{l}\text { Consumo o } \\
\text { total energético }\end{array}$ & $\begin{array}{l}\text { Existencia de un plan de ahorra energético } \\
\text { Consumo o total de energía por empleado }\end{array}$ \\
\hline
\end{tabular}


Para evaluar los indicadores de sostenibilidad se elaboró una encuesta que permitiera recolectar los datos necesarios para su medición tanto de los factores económicos, sociales y medio ambientales.

Posteriormente, se procedió a pasar las encuestas a cada una de las empresas que integran la Asociación de Comerciantes de pupusas de Arroz El Manguito de Olocuilta (ACPAMOL).

Para algunos indicadores fue necesario solicitar documentación con el fin de extraer datos específicos para la medición de algunos indicadores, estos documentos se detallan en la Tabla 2.

\begin{tabular}{|c|l|}
\hline CATEGORIA & \multicolumn{1}{|c|}{ DOCUMENTO } \\
\hline ECONÓMICO & $\begin{array}{l}\text { Balance general } \\
\text { Estado de resultados }\end{array}$ \\
\hline SOCIAL & $\begin{array}{l}\text { Reporte de accidentes } \\
\text { Planilla de personal } \\
\text { Reporte de conflictos reportados } \\
\text { Reporte de quejas de clientes } \\
\text { Reporte de rotación de personal }\end{array}$ \\
\hline AMBIENTAL & $\begin{array}{l}\text { Recibo de agua potable } \\
\text { Recibo de energia eléctrica }\end{array}$ \\
\hline
\end{tabular}

Una vez se terminaron de completar todas las encuestas se procedió a procesar la información en una hoja de cálculo con el propósito de aplicar de forma estandarizada las respectivas fórmulas de todos los indicadores.

Para otorgar una calificación a cada uno de los indicadores y subindicadores se partió de un punto en común que permitiría dar un diagnóstico de sostenibilidad más preciso, en donde se otorgaba al total de indicadores una calificación de $100 \%$, es decir, que los 11 indicadores a evaluar (donde se exceptúa el indicador "consumo de agua") obtuvieron una ponderación de 9.0909\%.

Las ponderaciones aplicadas a los resultados se presentan en la Figura1.

La medición de indicadores vinculados a preguntas con respuestas dicotómicas (sí o no), se realizó considerando la respuesta y su metodología de cumplimiento, dada la complejidad de lo que se pretende evaluar.
Por lo que si se obtenía como respuesta "Sí" y cumplía con la metodología entonces se le asignaba un $100 \%$ de la ponderación. En el caso que la respuesta fuese "Sí" pero no cumplía con la metodología este únicamente obtendría el $50 \%$ de la ponderación.

\section{Casos especiales:}

El subindicador "Salario mínimo de la empresa en comparación al de ley" entraba en la categoría mencionada, dado que pretendía evaluar si se otorgaba un salario acorde a la ley, independientemente del valor numérico.

El subindicador "Porcentaje de personal capacitado" se evalúo de esta forma dado que se presentó únicamente el caso en que todos estuviesen capacitados o ninguno de ellos.

Las preguntas en las cuales se obtenía como respuesta un porcentaje (\%) se evaluaron multiplicando el valor obtenido por la ponderación correspondiente al subindicador, con lo que se obtuvo la porción correspondiente de la calificación.
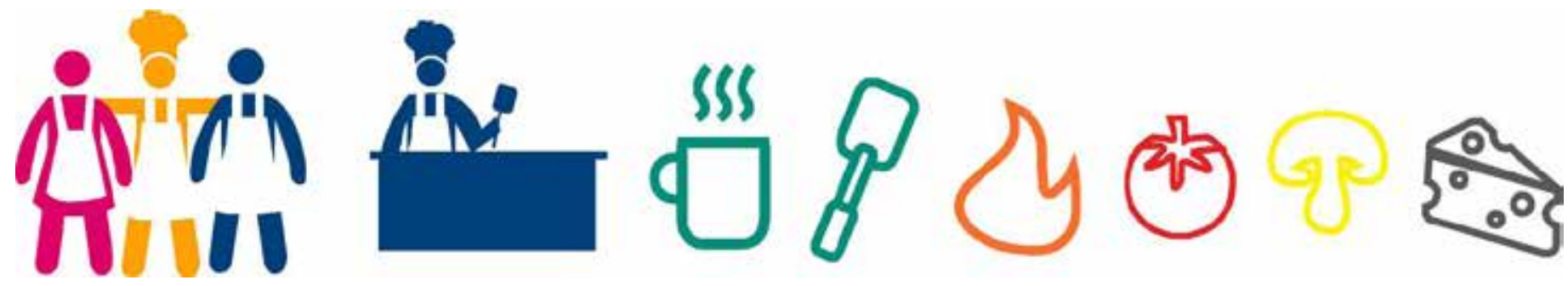


\section{Indicadores económicos}

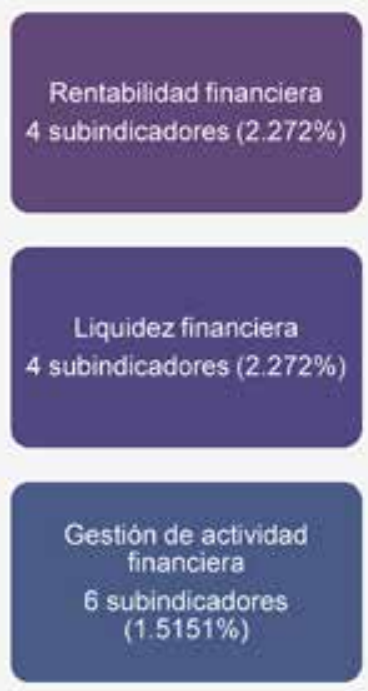

\section{Indicadores sociales}

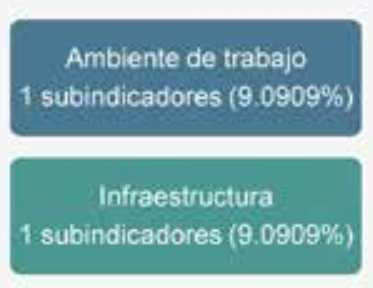

Relación con el trabajador

7 subindicadores (1:2987\%)

Gestión de relación con

proveedores y clientes

4 subindicadores $(2.272 \%)$

Calidad de productos y servicios

3 subindicadores (3.0303\%)

\section{Indicadores ambientales}

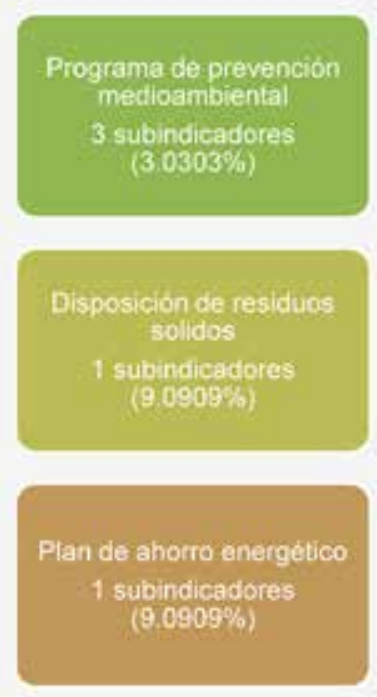

Figura1. Ponderación (porcentual) de subindicadores.

Fuente: Elaboración propia.

Para las preguntas en las cuales se obtenía un valor numérico tal como en el indicador "Consumo de agua" y en los subindicadores "Consumo de energía por empleado" y "Total de residuos producidos por la empresa", fueron evaluados únicamente de forma comparativa presentando razones entre variables, dado que no existía ningún valor óptimo previamente establecido. Por tal motivo, estos no contaban con una ponderación.

Para los indicadores financieros se determinaron una serie de valores, los cuales serían la línea base de ratios para los resultados que se obtuviesen al evaluarlos en los parámetros. Estos permitieron determinar si la empresa contaba con índices financieros aceptables o no, otorgando así respuestas cerradas de "Sí" y "No". En este caso, si se obtenía como resultado "Sí" se le asignaba directamente el $100 \%$ de la ponderación.

\section{Resultados y discusión}

Los resultados fueron obtenidos de acuerdo con cada uno de los factores evaluados dentro de la investigación.

\section{A. Indicadores económicos}

El factor económico a nivel general fue evaluado a partir de tres indicadores:

1. Rentabilidad financiera: pretende controlar los gastos de la empresa para así obtener las ventas que se transformen en utilidades para la empresa.

2. Liquidez financiera: permite analizar la capacidad con la que cuentan los negocios para cumplir con sus obligaciones.

3. Gestión de actividad financiera: permite medir la eficiencia con la que la empresa utiliza sus activos respecto a su ciclo productivo.

En los tres indicadores las 23 empresas en estudio obtuvieron el $100 \%$ de la calificación.
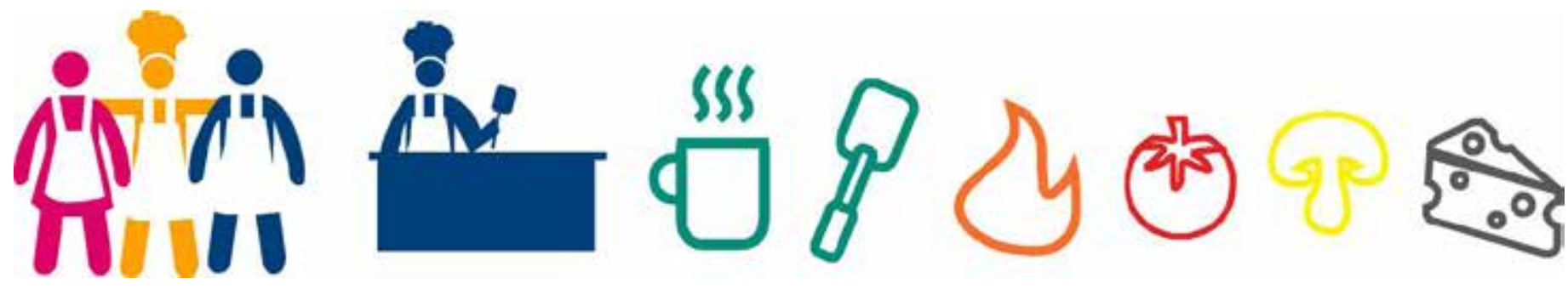


\section{B. Indicadores sociales}

El factor social a nivel general fue evaluado a partir de cinco indicadores:

1. Ambiente de trabajo: pretende evaluar el ambiente laboral bajo el cual el empleado realiza sus actividades diarias. Solamente tres empresas cumplieron con el $100 \%$ de la calificación posible, lo cual significa que cumplieron con el requisito principal de poseer un programa de prevención de riesgos ocupacionales.

2. Infraestructura: busca medir la calidad de la infraestructura con la que cuentan las instalaciones. Todas las empresas cumplieron con la mayor nota posible. Sin embargo, debido a que no emplean ningún tipo de metodología de evaluación o análisis, la calificación se redujo a la mitad, evidenciando de esa forma que los casos de accidentes son poco frecuentes o nulos a pesar de no tener un plan de contingencia para evitar 0 reducir el riesgo.

3. Relación con el trabajador: pretende evaluar las condiciones en las cuales el empleado desarroIla su trabajo. Únicamente tres establecimientos destacaron obteniendo el puntaje más alto respecto a las demás; sin embargo, ninguna empresa obtuvo la mayor calificación posible debido, principalmente, al subindicador de Rotación de Personal y Prestaciones Sociales de acuerdo con la Ley.

4. Gestión de relación con proveedores y clientes: tiene como fin determinar la forma en la que se está trabajando con los proveedores y los clientes. El $82 \%$ del total de pupuserías obtuvo la mayor nota posible del indicador, principalmente a raíz que, si contaban con un sistema de comunicación con el cliente. Esto demuestra que la mayoría de las pupuserías gestionan de manera correcta su relación con clientes y proveedores.

5. Calidad de productos y servicios: pretende asegurar el nivel de calidad tanto del producto como del servicio que brinda la empresa. El $21 \%$ de los negocios obtuvieron la mayor nota respecto a las demás, principalmente porque cumplieron con el subindicador del plan de mejora continua, un 52\% obtuvieran una nota media y $26 \%$ no lograron obtener puntaje alguno en ningún subindicador.

\section{Indicadores ambientales}

El factor ambiental fue evaluado a partir de tres indicadores:

1. Programa de gestión medioambiental: pretende validar la existencia de metodologías que se lleven a cabo con el fin de hacer conciencia para eliminar las consecuencias negativas que sus procesos productivos provocan al medio ambiente. El $39 \%$ de las 23 empresas destacaron con una calificación mayor en comparación con el porcentaje restante debido, principalmente, a que cumplieron con el subindicador de tener conocimiento respecto al tema medioambiental, evidenciando de esta forma que a nivel general no cuentan con programas que hagan conciencia respecto a los efectos negativos que pueden provocar las acciones cotidianas que realizan para el desarrollo del negocio.

2. Consumo de agua: este indicador no fue medido con un parámetro como tal, por lo que únicamente se determinó el valor promedio del consumo de agua (m3) respecto a 10,100 pupusas. Evidenciando que no todos los negocios tenían un gasto similar. El valor medio es de $26.96 \mathrm{~m} 3$ con una desviación estándar de $19.74 \mathrm{~m}^{3}$

3. Consumo total energético: pretende medir el nivel de consumo eléctrico de cada una de las empresas con el fin de conocer la dinámica de gasto y justificar la razón por la que se utiliza esa cantidad de energía. El resultado se midió con el subindicador: Existencia de un plan de ahorro anergético, donde el $74 \%$ de las 23 empresas cumplen con este requisito tomando medidas que les permitan ahorrar energía por lo que obtuvieron la mayor calificación posible, el $26 \%$ restante de los negocios no implementan ninguna medida para poder controlar sus gastos de energía.

Sin embargo, para este indicador también se utilizó un subindicador de consumo de kilovatios por hora, obteniéndose un valor medio es de $171.70 \mathrm{~kW} / \mathrm{h}$ con una desviación estándar de $99 \mathrm{~kW} / \mathrm{h}$. 
La Figura 2 muestra los valores promedio para los subindicadores de sostenibilidad para las empresas pertenecientes al pupusódromo "El Manguito" medido cada uno en una escala de 0 a 100, siendo 100 la mayor calificación para el subindicador.

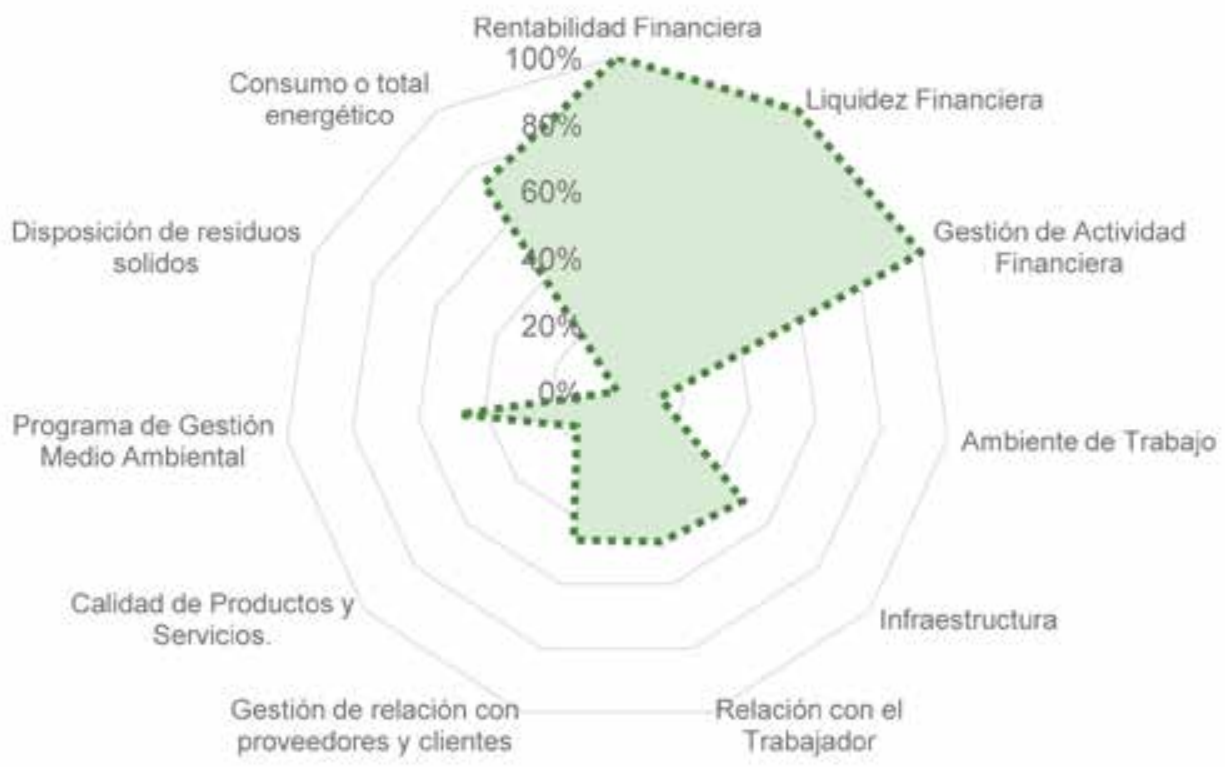

Figura 2. Valor promedio de los subindicadores de sostenibilidad. Fuente: Elaboración propia.

A partir de los resultados obtenidos por cada uno de los indicadores se pudo determinar el nivel de sostenibilidad total obtenido por cada una de las empresas. De forma general se puede establecer que las empresas cuentan con un nivel de sostenibilidad promedio de $53.83 \%$ con una desviación estándar de $4.06 \%$, siendo el valor mínimo obtenido de 42.64 \% y máximo de $57.84 \%$

Evidentemente, la mayor calificación resulta ser deficiente, ya que no existe un equilibrio de los factores económicos, medio ambientales y sociales que integran el nivel de sostenibilidad de una empresa, por lo cual el diagnóstico general de sostenibilidad del pupusódromo "El Manguito" es de bajo rendimiento. Sin embargo, este escenario permite la identificación precisa y estratégica de una gama de oportunidades de mejora que generarían beneficios importantes en los negocios en relación con la sostenibilidad.

\section{Conclusiones}

El desarrollo sostenible en El Salvador es un tema que ha venido a tomar relevancia en los últimos años, tanto en el sector de comercio como en el sector de servicios ya que busca un bienestar total, equilibrado tanto para clientes, empleados y empleadores. La investigación realizada permitió conocer la situación actual en cuanto al desarrollo sostenible de las micro y pequeñas empresas dedicadas a la venta de pupusas en Olocuilta, determinando así que a pesar de los resultados de los indicadores propuestos la variación entre las puntuaciones de las pupuserías no fue tan grande, quedando en evidencia que el tema del desarrollo sostenible es un concepto nuevo para las micro y pequeñas empresas pertenecientes a la muestra estudiada especialmente en los factores sociales y medio ambientales los cuales afectaron en gran parte la calificación final de cada una.

Muchos de los indicadores propuestos al ser evaluados en cada una de las pupuserías, parecían lograr el objetivo general del mismo. Sin embargo, estos en su mayoría no cumplían con la sistematización requerida. Esto demuestra que no cuentan con un proceso definido para lograr las metas en el tema de sostenibilidad, por lo que se ha determinado que es necesario que las empresas tengan un mayor control de los puntos deficientes identificados, tomando un control más preciso en las mejoras para generar y almacenar información histórica de los eventos, así identificar tendencias y poder tomar las medidas de control necesarias para evitar caer en el incumplimiento del indicador. 
A nivel medio ambiental, se encontraron limitaciones en cuanto a la existencia de parámetros óptimos con los cuales comparar la situación actual de las pupuserías. Dicho escenario se registra en el consumo del agua y el de energía eléctrica por lo que se realizó un análisis global de los parámetros de consumo. En relación al consumo de agua, siendo un insumo directo para la producción, se evidenció una variabilidad notable en la cantidad de agua empleada por cada 10,100 pupusas, por lo que se determina que aquellas pupuserías las cuales tienen un valor elevado en relación a los valores menores evidenciados tienen la oportunidad de optimizar este recurso ya que se demuestra que es posible producir con menor cantidad de agua. En el consumo de energía eléctrica, siendo un insumo no directo en la producción, se registra una variabilidad notable el cual implica un aumento de costos sin impacto en la producción, por lo que reducir dicho valor con ayuda de medidas o planes de gestión de recursos contribuiría a mejorar la sostenibilidad de las empresas y mejorar las utilidades percibidas.

\section{Bibliografia:}

Alas Henríquez, C. R., Avilés Aguilar, C. E., \& Burgos Villatoro, S. A. (2018). IDENTIFICACIÓN DE Indicadores que Caracterizan una micro y pequeña empresa sostenible en El Salvador. Antiguo Cuscatlan: Universidad Centroamerica José Simeón Cañas. Alcaldía Municipal de Olocuilta. (11 de Noviembre de 2011). Alcaldía Municipal de Olocuilta. Recuperado el 4 de Mayo de 2019, de http://www.olocuilta.gob.sv/index.php/el-municipio/turismo/19-turismo-gastronomico Alvarez, M. (Diciembre de 2009). Comisión Económica para América Latina y el Caribe. Obtenido de https://repositorio.cepal.org/ bitstream/handle/11362/2022/1/Manual_Micro_Pequenha_Mediana_Empresa_es.pdf

Bermúdez Cardona, C. M., Portillo Granados, T. E., \& Henríquez Mejía, H. A. (Noviembre de 2016). Identificación de las competencias empresariales y su incidencia en la rentabilidad y sostenibilidad en las micro y pequeñas (MYPES) de la zona urbana de los municipios de Coatepeque y San Rafael Cedros del departamento de Cuscatlán. San Salvador: Universidad de El Salvador.

Obtenido de http://ri.ues.edu.sv/10111/1/Competencias\%20empresariales\%20en\%20las\%20MYPES.pdf Comisión Económica para América Latina y el Caribe (CEPAL). (octubre de 2004).

Desarrollo de Infraestructura y crecimiento económico: Revisión conceptual. Obtenido de https://repositorio.cepal.org/bitstream/handle/11362/6441/1/S048642_es.pdf CONAMYPE. (2014). Comisión Nacional de la Micro y Pequeña Empresa.

Obtenido de https://www.conamype.gob.sv/wp-content/uploads/2013/04/Ley-MYPE-web.pdf

Gil Lafuente, A. M., \& Paula, L. B. (2011). La gestión de los grupos de interés. Revista de Métodos

Cuantitativos para la Economía y la Empresa, 71-90. Gómez, C. (2014). El desarrollo sostenible: conceptos básicos, alcance y criterios para su evaluación. Cambio climático y desarrollo sostenible: Bases conceptuales para la educación en Cuba, 90-111. Gualdrón, M. E. (2018). Perfil de Sostenibilidad Empresarial de Micros Pequeñas y Medianas Empresas.

Universidad Nacional Abierta y Distancia UNAD, Bucaramanga, Colombia. Recuperado el 22 de Abril de 2019, de https://stadium.unad.edu.co/preview/UNAD.php?url=/bitstream/10596/19489/1/63439978.pdf

Menéndez, F. (2018). Plan de Negocio de pupusa de Olocuilta. Morillo, M. (Enero de 2001). Rentabilidad Financiera y Reducción de Costos. Obtenido de https://www.redalyc.org/html/257/25700404/ ONU. (1999). Pacto Mundial de las Naciones Unidas. 10 Principios.

Recuperado el 24 de Abril de 2019, de https://www.pactomundial.org/category/aprendizaje/10-principios/ Organización Internacional del Trabajo. (Octubre de 2012). El desafío de la promoción de Empresas Sostenibles en América Latina y el Caribe: un análisis regional comparativo.

Obtenido de https://www.ilo.org/wcmsp5/groups/public/---americas/---ro-lima/documents/publication/wcms_205234.pdf Saavedra G., M. L. (2008). Caracterización e importancia de las MIPYMES en Latinoamerica: Un estudio comporativo. Actualidad Contable Faces, 122-134.

Sumelzo, N. S. (2012). La Sostenibilidad en el Sector Empresarial. UPC Universitat Politècnica, Catalunya. Recuperado el 20 de Abril de 2019, de https://upcommons.upc.edu/bitstream/handle/2099.1/18820/TFM_NSanchez_La\%20sostenibilidad\%20en\%20el\%20sector\%20empresarial.pdf Universidad de Barcelona. (2011). Reflexión sobre los desafíos a los que se enfrentan las empresas en la búsqueda de la sostenibilidad empresarial. Obtenido de https://www.econstor.eu/bitstream/10419/59090/1/684025647.pdf Vilches, A., Pérez, D. G., Toscano Grimaldi, J. C., \& Macías Álvarado, Ó. (2014). La sostenibilidad o sustentabilidad como revolución cultural. tecnocientífica y política. Obtenido de https://www.oei.es/historico/decada/ accion.php?accion=1 Zendesk. (2016). Entrepeneur. Obtenido de https://www.entrepreneur.com/article/272461 